\title{
Effect of clinical guidelines in nursing, midwifery, and the therapies: a systematic review of evaluations
}

\author{
L H Thomas, E McColl, N Cullum, N Rousseau, J Soutter, N Steen
}

Centre for Health Services Research, School of Health Sciences, 21 Claremont Place, University of Newcastle upon Tyne, UK

L H Thomas, research associate

E McColl, senior research associate $\mathrm{N}$ Rousseau, research associate

J Soutter, research associate

N Steen, research associate

Centre for

Evidence-Based

Nursing, Department of Health Studies, University of York, UK $\mathrm{N}$ Cullum, reader

Correspondence to: Dr LH Thomas, Centre for Health Services Research, University of Newcastle, 21 Claremont Place, Newcastle upon Tyne NE2 4AA, UK. Telephone 0044191222 7047; fax 0044191222 6043; email

L.H.Thomas@ncl.ac.uk

Accepted for publication 23 October 1998

\begin{abstract}
Background-Although nursing, midwifery, and professions allied to medicine are increasingly using clinical guidelines to reduce inappropriate variations in practice and ensure higher quality care, there have been no rigorous overviews of their effectiveness. 18 evaluations of guidelines were identified that meet Cochrane criteria for scientific rigor.

Methods-Guideline evaluations conducted since 1975 which used a randomised controlled trial, controlled before and after, or interrupted time series design were identified through a combination of database and hand searching.

Results-18 studies met the inclusion criteria. Three studies evaluated guideline dissemination or implementation strategies, nine compared use of a guideline with a no guideline state; six studies examined skill substitution: performance of nurses operating according to a guideline were compared with standard care, generally provided by a physician. Significant changes in the process of care were found in six out of eight studies measuring process and in which guidelines were expected to have a positive impact on performance. In seven of the nine studies measuring outcomes of care, significant differences in favour of the intervention group were found. Skill substitution studies generally supported the hypothesis of no difference between protocol driven by nurses and care by a physician. Only one study included a formal economic evaluation, with equivocal findings.

Conclusions-Findings from the review provide some evidence that care driven by a guideline can be effective in changing the process and outcome of care. However, many studies fell short of the criteria of the Cochrane Effective Practice and Organisation of Care Group (EPOC) for methodological quality.

(Quality in Health Care 1998;7:183-191)
\end{abstract}

Keywords: clinical guidelines; nursing; midwifery; systematic review; clinical effectiveness

\section{Introduction}

Clinical guidelines in medicine have been shown to improve the performance of doctors and to promote the delivery of high quality, evidence-based health care. ${ }^{12}$ The benefits of developing and implementing guidelines may not, however, be unique to this profession. In nursing, midwifery, and professions allied to medicine, there is increasing interest in the use of guidelines to reduce inappropriate variations in practice and ensure higher quality care..$^{3-6}$

Preliminary searches indicated little formal evaluation of the effectiveness of the implementation strategies adopted by nurses, ${ }^{5}$ midwives, and professions allied to medicine. In the United States, guideline dissemination seems to be through massive distribution to professional and lay groups, ${ }^{5}$ although there is evidence from the medical profession to suggest that dissemination by publication or direct mailing are the least successful strategies. ${ }^{7-11}$ However, it cannot be assumed that those strategies proved to work for doctors will transfer directly to other groups of healthcare professionals with a dissimilar educational preparation and a different management structure.

We therefore undertook a systematic review of evaluations of clinical guidelines in nursing, midwifery, and professions allied to medicine. ${ }^{12}$ This was registered with the Cochrane Effective Practice and Organisation of Care Group (EPOC; formerly the Cochrane Collaboration on Effective Professional Practice).

Our review had two main objectives.

(1) To identify evaluations (in any setting) of clinical practice guidelines and of related dissemination and implementation strategies in nursing, midwifery, health visiting, and other professions allied to medicine-podiatry, speech and language therapy, physiotherapy, occupational therapy, dietetics, clinical psychology, pharmacy, and radiography.

(2) To derive estimates of the effectiveness and cost effectiveness of clinical practice guidelines as strategies for promoting improved professional practice and enhanced patient outcomes in nursing and professions allied to medicine.

We sought, as far as possible, to replicate the medically oriented work of Grimshaw et al. ${ }^{12}{ }^{13}$ We wished to describe the nature of guidelines for the target professions. (Who developed them; were they derived by consensus methods; were they evidence-based?) We also wanted to investigate whether guideline dissemination and implementation strategies found to be effective for doctors were transferable to nursing and other professions allied to medicine. However, our review was intended to be primarily descriptive, rather than driven by a hypothesis. 


\section{Methods}

INCLUSION AND EXCLUSION CRITERIA

The definition of clinical guidelines by Field and Lohr ${ }^{14}$ as "systematically developed statements to assist practitioner decisions about appropriate health care for specific clinical circumstances" was used. We were interested in all types of guideline, not simply those based on evidence.

To be eligible for inclusion in our review, a study had to be an evaluation of the effect of a clinical guideline on the behaviour of nurses or other professionals allied to medicine or on patient outcomes. We included studies which evaluated an entire, or identified component, of a guideline aimed at these professions and which specified which part or parts of the guideline should be implemented by the profession in question. We considered not only studies of guidelines developed by the target profession for their own use, but also those in which the guideline was developed or disseminated by another professional group but targeted the behaviour of nurses or other professionals allied to medicine.

We included both studies which evaluated the effectiveness of guidelines against a nonintervention control (routine care) and those comparing different dissemination and implementation strategies of a guideline - for example, dissemination strategy $\mathrm{X}$ versus dissemination strategy Y. Studies which simply described the development, dissemination, or implementation of a guideline, without an evaluation of the impact, were excluded, as were studies in which $<50 \%$ of recipients of the guideline were nurses, midwives, or other professions allied to medicine and studies in which it was not possible to separate the effects of guidelines on the behaviour of professionals from the impact on the behaviour of other health professionals. A start date of 1975 was imposed, firstly to make our review comparable with that of Grimshaw and Russell, ${ }^{12}$ and secondly because MEDLINE does not contain abstracts before that year.

METHODOLOGICAL CRITERIA

To be compatible with Grimshaw et al, ${ }^{12}{ }^{13}$ and in line with standard practice for EPOC reviews, three types of study designs were included.

(1) Randomised controlled trials, where we distinguished between simple randomised controlled trials (in which the unit of allocation is the patient) and designs in which the unit of allocation is the health professional and which are therefore less susceptible to contamination. (2) Controlled before and after studies.

(3) Interrupted time series designs, if there was a clearly defined point in time when the intervention occurred and at least two data collection points before and two after the intervention.

SYSTEMATIC REVIEW OF LITERATURE

We searched: (a) computerised databases (MEDLINE; CINAHL (Cumulative Index of Nursing and Allied Health Literature); PsycLIT; EMBASE; NEED (NHS Economic
Evaluations Database); DARE (Database of Abstracts of Reviews of Effectiveness); DHSSDATA; SIGLE; and the National Research Register); (b) the EPOC register of studies; (c) citations in papers identified, and (d) Quality in Health Care ${ }^{\star}$. We also contacted content area experts, the libraries of appropriate professional bodies - for example, the Royal College of Nursing - and various mailbases on the internet-for example, evidence-basedhealth@mailbase.ac.uk.

Search strategies for electronic databases were developed sequentially, starting with MEDLINE, as this was expected to yield the highest number of relevant papers. We adapted the focus section of the EPOC search strategy (designed to identify randomised trials, controlled before and after and interrupted time series studies) to expand the section on clinical guidelines. We searched for guidelines and various synonyms in titles and abstracts as well as carrying out keyword searches. MEDLINE also identifies "publication types", which allowed us to search for papers tagged as "practice guideline" or "guideline". We then worked with an information scientist to adapt the search strategy for use with the other databases.

SCREENING OF IDENTIFIED PAPERS

The full text version of each study identified as potentially relevant to our review was read by one reviewer (NC, EM, NR, JS, or LHT) and assessed against several criteria with a decision tree developed for this review. To establish consistency between raters, all reviewers initially completed checklists for the same five papers; discrepancies were discussed and resolved. All those studies identified by one reviewer as meeting all the inclusion criteria, and those in which there was an element of doubt, were referred to a second reviewer; discussion between the two reviewers took place to resolve any remaining differences.

DATA EXTRACTION

We used the checklist and data extraction template developed by EPOC to abstract data from studies meeting our inclusion criteria. Data extraction was undertaken by two reviewers working independently and included both methodological details - for example, units of allocation and data analysis; methodological quality criteria - and information about study participants, interventions, outcomes, and results. The two reviewers then met to compare their judgements and any discrepancies were discussed and resolved. A "master version" of the data extraction template was agreed and the details were put into an Idealis $\mathrm{t}^{15}$ database.

* Quality in Health Care is now included in the following databases: HEALTH (Health Planning and Administration) Database of the National Library of Medicine and American Hospital Association Resource Center from 1995 onwards, CINAHL, ISI Science Citation Index (public health, environmental and occupational), ISI Current Contents (clinical medicine), and EMBASE. 
DATA ANALYSIS

As the heterogeneity of clinical areas, design of studies, source and format of interventions, outcomes measured, and participating health professionals, was substantial, an overall estimate of effect was likely to have little practical meaning. We therefore opted simply to report the effects on the process and outcome of care in the same way as they were reported in the original papers, rather than to attempt comparisons with some common unit of change.

\section{Results}

We identified 18 studies which met our inclusion criteria. In all but one, guidelines were aimed, in part at least, at nurses. Of these studies, in five cases, ${ }^{16-20}$ physicians were also targeted. The study by Franz et $a l^{11}$ was aimed solely at dietitians. Shaffer and Wexler ${ }^{22}$ presented a truly multi-disciplinary guideline, aimed at an entire lipid clinic team comprising nurses, pharmacists, psychologists, and dietitians.

METHODOLOGICAL QUALITY

We identified 13 randomised controlled trials. In three studies the unit of allocation was the health professional or provider unit. ${ }^{16}{ }^{17}$ In all three the randomisation process was not described and it is not clear whether there was blinded assessment of outcomes. One trial ${ }^{16}$ had a unit of analysis error: the unit of randomisation was the provider, but the unit of analysis was the patient. Such unit of analysis errors artificially increase the power of the study and may lead to misleadingly significant findings.

There were 10 randomised controlled trials in which the unit of allocation was the patient. ${ }^{18} 19{ }^{2124-30}$ Six of these p $^{1825-28}$ examined the effect of substituting another health professional (usually a nurse) working to protocols for usual care provided by a doctor: obviously in these studies, randomisation by health professional would not be possible. There were major differences in methodological quality: all 13 studies had good follow up; however, just over half (7/13) blindly assessed outcomes and reliably measured at least some key outcomes (7/13). A few studies (4/13) reported method of randomisation.

We identified two controlled before and after studies where a second site acted as the control. ${ }^{2231}$ In the study of Larson and Hargiss, ${ }^{31}$ five units in a single hospital participated; two received the intervention and three served as controls. No data were presented about the comparability of intervention and control sites; baseline measurement showed significant differences on one variable likely to influence differences after intervention (accuracy of recording of last intravenous infusion). At least some outcomes were measured reliably but none were assessed blindly. Protection against contamination seemed adequate. In the study by Shaffer and Wexler, ${ }^{22}$ two clinics (one intervention, one control) within the same medical centre participated. No data were presented on the similarity of the clinics; patients selected for the study from each clinic were matched according to age and cholesterol concentration. Outcomes measured were reliable and were assessed blindly. As the lipid clinic team worked only in the intervention clinic, it is likely that there was successful protection against contamination between clinics.

Three studies were interrupted time series designs..$^{20} 3233$ In the study by French et $a l^{20}$ the intervention seemed to be independent of other changes during the study period; in the study of Mitchell and Jones ${ }^{32}$ no details were provided as to whether this was or was not the case. In the study of Tilden and Shepherd, ${ }^{33}$ in which the targeted behaviour was identification of battered women, the authors stated that media coverage and an initiative by hospital administration (aimed at all staff) to increase the level of detail in patient documentation may have intensified the effect of the intervention. No interrupted time series study had $\geqslant 12$ data points before and after the intervention; only one study ${ }^{20}$ did a formal test for trend. All interrupted time series studies were prone to detection bias. In two studies ${ }^{20}{ }^{33}$ data collection methods were identical before and after the intervention. However, in the study of Mitchell and Jones ${ }^{32}$ data on rates of falls before intervention were collected from retrospective analysis of incident forms of falls, and prospective data were collected by adding a "falls reporting document" to the incident form. As this seemed to be part of the intervention, it directly influenced data collection. Whether the intervention directly influenced data collection in the two other interrupted time series studies is not clear. Outcomes were not assessed blindly in one study ${ }^{32}$; in the remaining two studies it is not clear whether this was the case. In only one study ${ }^{33}$ was information provided about the completeness of the data set: all adult female trauma patients were included. No interrupted time series study measured outcomes reliably.

\section{STUDIES EVALUATING DISSEMINATION AND} IMPLEMENTATION STRATEGIES

Three studies (table) ${ }^{16}{ }^{17} 23$ explicitly evaluated guideline dissemination or implementation strategies. All were randomised controlled trials. Within these studies, the interventions were heterogeneous, precluding combining the findings from two or more studies. Seto et $a l^{23}$ in their evaluation of opinion leaders and in-service lectures as methods of disseminating a guideline on urinary catheter care to nurses, compared three groups: all received guidelines; one received both lectures and input from local opinion leaders; the remaining two received either lectures or input from opinion leaders. Some evidence was found that the combined strategy of opinion leaders and lectures led to higher compliance with correct practice than did either strategy alone. The weakest strategy was a lecture alone.

Herman et $a l^{16}$ found that a "nurse protocol" intervention (primarily an implementation strategy) led to positive changes in the process of care (offer of influenza and pneumococcal vaccine). There were three comparison groups: all received guidelines, educational materials, 


\begin{tabular}{|c|c|c|c|c|c|}
\hline $\begin{array}{l}\text { Study and } \\
\text { study design }\end{array}$ & Participants & Clinical area & $\begin{array}{l}\text { Results } \\
\text { comparisons }\end{array}$ & Main process effect & Main patient outcomes \\
\hline \multicolumn{6}{|c|}{ Dissemination and implementation strategies: } \\
\hline $\begin{array}{l}\text { Herman et } \\
\text { al1994 } \\
\text { RCT-Pr US }\end{array}$ & $\begin{array}{l}\text { Nurses (number } \\
\text { not specified) and } \\
66 \text { doctors in } 3 \\
\text { ambulatory } \\
\text { medical practices } \\
\text { Provider: } 1 \\
\text { practice in each of } \\
3 \text { arms Proportion } \\
\text { of patients } \\
\text { followed up: } \\
100 \% \text { of eligible } \\
\text { patients for } \\
\text { influenza } \\
\text { vaccination group; } \\
75 \% \text { for } \\
\text { pneumococcal } \\
\text { vaccination group }\end{array}$ & $\begin{array}{l}\text { Pneumococcal } \\
\text { and influenza } \\
\text { vaccinations }\end{array}$ & $\begin{array}{l}\text { I1: Guidelines; } \\
\text { educational } \\
\text { materials; lectures } \\
\text { I2: As 1; provider } \\
\text { and patient } \\
\text { education } \\
\text { provided at each } \\
\text { clinic visit I3: As } \\
\text { 1; plus protocol }\end{array}$ & $\begin{array}{l}\text { Offer of influenza vaccination: I1: } 48 \% \\
(129 / 271) \mathrm{I} 2: 50 \%(122 / 242) \mathrm{I} 3: 68 \% \\
(166 / 243) \mathrm{p}=0.006 \text { Offer of } \\
\text { pneumococcal vaccination: I1: } 5.4 \% \\
(16 / 295) \mathrm{I} 2: 6.5 \%(19 / 292) \mathrm{I} 3: 28.3 \% \\
(89 / 314) \mathrm{p}=0.001\end{array}$ & $\begin{array}{l}\text { Influenza vaccination: } \mathrm{I} 1: 5.9 \% \text {; } 2: 5.8 \% \text {; I3 } \\
13.2 \% \mathrm{p}=0.003 \text { Pneumococcal vaccination } \\
\mathrm{I} 1: 2.0 \% ; \mathrm{I} 2: 1.4 \% ; \mathrm{I} 3: 6.7 \% \mathrm{p}<0.001\end{array}$ \\
\hline $\begin{array}{l}\text { McDonald } \\
\text { et al } 1980^{17} \\
\text { RCT-Pr } \\
\text { (Crossover } \\
\text { trial) US }\end{array}$ & $\begin{array}{l}5 \text { Nurses and } 26 \\
\text { doctors in } 1 \\
\text { medical } \\
\text { outpatient clinic } \\
\text { 3-Treatment } \\
\text { crossover design: } \\
\text { all providers } \\
\text { experienced all } \\
\text { conditions in } 1 \text { of } \\
6 \text { possible } \\
\text { different } \\
\text { treatment } \\
\text { sequences } \\
\text { Proportion of } \\
\text { patients followed } \\
\text { up: not applicable }\end{array}$ & $\begin{array}{l}\text { Use and } \\
\text { follow up of } \\
\text { medications }\end{array}$ & $\begin{array}{l}\text { I1: Computer } \\
\text { reminders I2: } \\
\text { Computer } \\
\text { reminders }+ \\
\text { bibliographic } \\
\text { citations C: } \\
\text { Standard practice }\end{array}$ & $\begin{array}{l}\text { Individual provider response rate }= \\
\text { number of appropriate responses/total } \\
\text { number possible: average nurse response } \\
\text { rate to prompts I1: } 30 \% \text {; }: 25 \%(n=5 \\
\text { nurses) Not significant: insufficient data } \\
\text { provided to calculate p values or } 95 \% \text { CIs }\end{array}$ & \\
\hline $\begin{array}{l}\text { Seto et al } \\
1991^{23} \\
\text { RCT-Pr } \\
\text { Hong Kong }\end{array}$ & $\begin{array}{l}220 \text { Nurses in } 1 \\
\text { hospital Provider: } \\
2 \text { wards in each of } \\
3 \text { arms Proportion } \\
\text { of patients } \\
\text { followed up: not } \\
\text { applicable }\end{array}$ & $\begin{array}{l}\text { Urinary } \\
\text { catheter care }\end{array}$ & $\begin{array}{l}\text { I1: Guidelines; } \\
\text { local opinion } \\
\text { leaders; lecture } \\
\text { I2: Guidelines; } \\
\text { local opinion } \\
\text { leaders C: } \\
\text { Guidelines; } \\
\text { lecture }\end{array}$ & $\begin{array}{l}\text { Change in reported practice, } 2 \text { weeks } \\
\text { before } v 5 \text { weeks after intervention: I1: } \\
5.63(50 \%) \text { I2: } 4.96(35 \%) \text { C: } 3.29(38 \%) \\
\text { Mean change in C significantly lower than } \\
\text { I1 and I } 2<0.05 \text { I } v \text { I } 2 \text { not significant: } \\
\text { insufficient data provided to calculate p } \\
\text { values or } 95 \% \text { CIs Percentage of correct } \\
\text { catheter practices, } 1 \text { month after } \\
\text { intervention: I1 } v \text { I2: p<0.05 I1 } v \text { C: } \\
\text { p }<0.05\end{array}$ & \\
\hline \multicolumn{6}{|c|}{ Comparison of guideline $v$ no guideline: } \\
\hline $\begin{array}{l}\text { Franz } \\
1995^{21} \\
\text { RCT-Pt US }\end{array}$ & $\begin{array}{l}\text { Dietitians } \\
\text { (number not } \\
\text { specified) in } \\
\text { outpatient clinics } \\
\text { Patients: } 247 \text { with } \\
\text { non-insulin } \\
\text { dependent } \\
\text { diabetes mellitus } \\
\text { Proportion of } \\
\text { patients followed } \\
\text { up: } 72 \% \text { in total }\end{array}$ & $\begin{array}{l}\text { Non-insulin } \\
\text { dependent } \\
\text { diabetes } \\
\text { mellitus }\end{array}$ & $\begin{array}{l}\text { I: Guidelines C: } \\
\text { Standard care }\end{array}$ & $\begin{array}{l}\text { Changes in treatment: I: } 18 \%(17 / 94) ; \mathrm{C} \text { : } \\
11 \%(9 / 85) \mathrm{p}=0.203\end{array}$ & 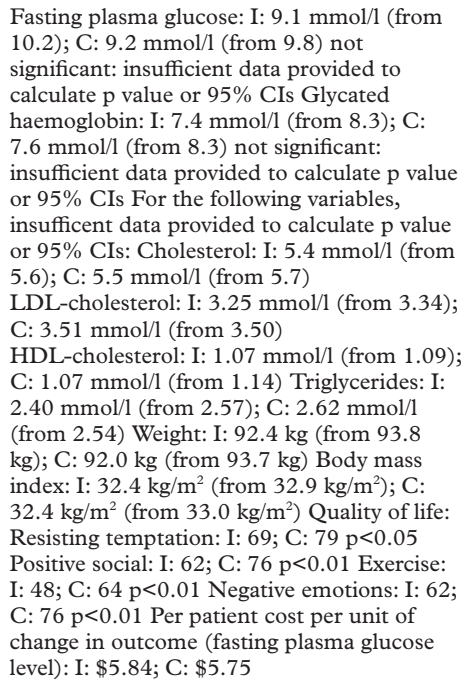 \\
\hline $\begin{array}{l}\text { Frigoletto et } \\
\text { al } 1995^{24} \\
\text { RCT-Pt US }\end{array}$ & $\begin{array}{l}\text { Two maternity } \\
\text { units in } 1 \text { hospital } \\
\text { Patients: } 1017 \\
\text { intervention } 917 \\
\text { control Protocol } \\
\text { eligible } \\
\text { sub-group: } 678 \\
\text { intervention } 585 \\
\text { control } \\
\text { Proportion of } \\
\text { patients followed } \\
\text { up: } 99 \% \text { in total }\end{array}$ & $\begin{array}{l}\text { Delivery of } \\
\text { first babies }\end{array}$ & $\begin{array}{l}\text { I: Protocol } \\
\text { (midwives) C: } \\
\text { Standard care }\end{array}$ & 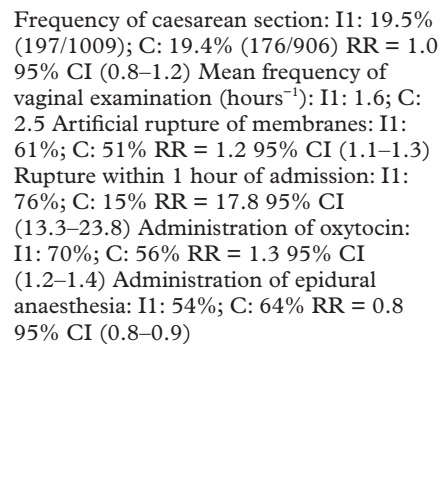 & $\begin{array}{l}\text { Rate of caesarean section: I1: } 19.5 \% \\
(197 / 1009) ; \mathrm{C}: 19.4 \%(176 / 906) \text { for all } \\
\text { patients RR }=1.095 \% \text { CI }(0.8-1.2) \text { I: } \\
10.9 \% \text { C: } 11.5 \% \text { for protocol eligible group } \\
\text { RR }=0.995 \% \text { CI }(0.4-1.9 \text { ) Duration of } \\
\text { labour (protocol eligible subgroup): I: } 6.2 \text {; C: } \\
8.9 \text { hours Insufficient data provided to } \\
\text { calculate } 95 \% \text { CIs Rate of maternal fever } \\
\text { (protocol eligible subgroup): I: } 7 \% \text {; }: 11 \% \\
\text { p=0.007 Rate of other maternal outcomes: } \\
\text { no significant differences in foetal distress, } \\
\text { placental abruption, shoulder dystocia, } \\
\text { vaginal lacerations Duration of labour }>12 \\
\text { hours (protocol eligible subgroup): I: } 9 \% \text {; C: } \\
26 \% \text { p }<0.0001 \text { Infant outcomes: no } \\
\text { significant differences in frequency of } \\
\text { jaundice, seizures, treatment for sepsis, } \\
\text { resuscitation at birth or admission to } \\
\text { neonatal ICU }\end{array}$ \\
\hline
\end{tabular}


Comparison tables (continued)

\begin{tabular}{|c|c|c|c|c|c|}
\hline $\begin{array}{l}\text { Study and } \\
\text { study design }\end{array}$ & Participants & Clinical area & $\begin{array}{l}\text { Results } \\
\text { comparisons }\end{array}$ & Main process effect & Main patient outcomes \\
\hline $\begin{array}{l}\text { Naylor } \\
1990^{29} \\
\text { RCT-Pt US }\end{array}$ & $\begin{array}{l}2 \text { Nurses (in } \\
\text { intervention } \\
\text { group) in } 1 \\
\text { hospital Patients: } \\
20 \text { intervention } 20 \\
\text { control } \\
\text { Proportion of } \\
\text { patients followed } \\
\text { up: } 100 \%\end{array}$ & $\begin{array}{l}\text { Discharge } \\
\text { planning for } \\
>70 \text { year-olds } \\
\text { in medical } \\
\text { and surgical } \\
\text { units }\end{array}$ & $\begin{array}{l}\text { I: Protocol C: } \\
\text { Standard practice }\end{array}$ & & $\begin{array}{l}\text { Mean duration of stay (days): I: } 8.2 \pm 4.9 \text {; C: } \\
9.05 \pm 7.66 \text { not significant: insufficient data } \\
\text { to calculate p value or } 95 \% \text { CIs } \\
\text { Postdischarge infection rates: I: } 33.3 \% \\
(6 / 20) ; \text { C: } 50 \%(10 / 20) \text { RR }=0.4395 \% \text { CI } \\
(0.11-1.57) \text { Postdischarge rates of } \\
\text { readmissions to hospital: I: } 16.7 \%(4 \\
\text { readmissions, } 3 \text { patients); C: } 64.7 \%(12 \\
\text { readmissions to hospital, } 11 \text { patients) p } \leqslant 0.05\end{array}$ \\
\hline $\begin{array}{l}\text { Naylor } \\
1994^{30} \\
\text { RCT-Pt US }\end{array}$ & $\begin{array}{l}2 \text { Nurses and } \\
\text { doctors (number } \\
\text { not specified) in } 1 \\
\text { hospital Patients: } \\
364 \text { patients } \\
\text { randomised; } 276 \\
\text { analysed: } 140 \\
\text { intervention } 136 \\
\text { control } \\
\text { Proportion of } \\
\text { patients followed } \\
\text { up: } 76 \% \text { in total }\end{array}$ & $\begin{array}{l}\text { Discharge } \\
\text { planning for } \\
>70 \text { year-olds } \\
\text { in medical } \\
\text { and surgical } \\
\text { units }\end{array}$ & $\begin{array}{l}\text { I: Protocol C: } \\
\text { Standard practice }\end{array}$ & & 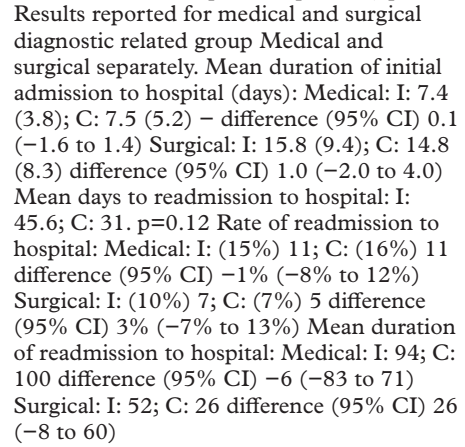 \\
\hline $\begin{array}{l}\text { Larson and } \\
\text { Hargiss } \\
1984^{31} \text { CBA } \\
\text { US }\end{array}$ & $\begin{array}{l}10 \text { nurses (in } \\
\text { intervention } \\
\text { group) and } \\
\text { doctors (number } \\
\text { not specified) in } 2 \\
\text { intervention and } 3 \\
\text { control wards in } 1 \\
\text { university } \\
\text { affiliated hospital } \\
\text { Patients: } 707 \text { (in } \\
\text { total) Proportion } \\
\text { of patients } \\
\text { followed up: not } \\
\text { clear }\end{array}$ & $\begin{array}{l}\text { Intravenous } \\
\text { therapy }\end{array}$ & $\begin{array}{l}\text { I: Guidelines; } \\
\text { outreach visits C: } \\
\text { Standard practice }\end{array}$ & $\begin{array}{l}\text { Percentage of items documented correctly: } \\
\text { I: increase from } 57 \% \text { to } 79 \% \text { C: increase } \\
\text { from } 52.5 \% \text { to } 68.5 \% ; \mathrm{p}<0.001 \text { Test } \\
\text { inappropriate }\end{array}$ & $\begin{array}{l}\text { Occurrence of iv related phlebitis: I: decrease } \\
\text { from } 33.5 \% \text { to } 20.9 \% p=0.05 \text { C: increase } \\
\text { from } 23.8 \% \text { to } 26.7 \% p=0.25 \text { Frequency of } \\
\text { bacterial colonisation of infusion devices: } \mathrm{I} \text { : } \\
\text { increase from } 12.7 \% \text { to } 19.4 \% p=0.25 \mathrm{C} \text { : } \\
\text { decrease from } 7.1 \% \text { to } 5.9 \% p=0.90 \text { Patient } \\
\text { comfort with IV insertion: not reported in } \\
\text { appropriate format }\end{array}$ \\
\hline $\begin{array}{l}\text { Shaffer and } \\
\text { Wexler } \\
1995^{22} \text { CBA } \\
\text { US }\end{array}$ & $\begin{array}{l}1 \text { lipid clinic team } \\
\text { (nurse led) and } 1 \\
\text { general medicine } \\
\text { clinic team in } 1 \\
\text { medical centre } \\
\text { Provider: } 1 \\
\text { intervention } 1 \\
\text { control Patients: } \\
60 \text { intervention } 60 \\
\text { control } \\
\text { Proportion of } \\
\text { patients followed } \\
\text { up: } 93 \% \\
\text { intervention } 88 \% \\
\text { control }\end{array}$ & $\begin{array}{l}\text { Hyper- } \\
\text { lipidaemia }\end{array}$ & $\begin{array}{l}\text { I: Guidelines } \\
\text { (nurse, clinical } \\
\text { pharmacist, } \\
\text { dietitian, clinical } \\
\text { psychologist) C: } \\
\text { No intervention } \\
\text { (doctors) }\end{array}$ & $\begin{array}{l}\text { Percentage of patients for whom a new } \\
\text { drug was prescribed: I: } 85 \%(51 / 60) \text {; C: } \\
18 \%(11 / 60) \text { p }<0.001 \text { Number of patients } \\
\text { in whom an existing drug was stopped: I: } \\
22 \text { patients; C: } 3 \text { patients. p<0.001 } \\
\text { Percentage of patients receiving expert } \\
\text { dietary counselling: I: } 100 \%(60 / 60) \text {; C: } \\
42 \%(25 / 60) \text { p<0.001 }\end{array}$ & 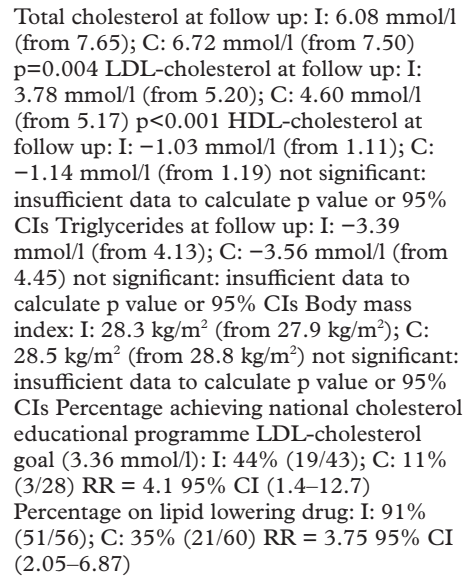 \\
\hline $\begin{array}{l}\text { French et al } \\
1989^{20} \text { ITS } \\
\text { Hong Kong }\end{array}$ & $\begin{array}{l}3 \text { Infection } \\
\text { control nurses in } \\
1 \text { hospital } \\
\text { Patients: ranged } \\
\text { from } 809 \text { to } 1260 \\
\text { in } 7 \text { prevalence } \\
\text { surveys }\end{array}$ & $\begin{array}{l}\text { Infection } \\
\text { rates }\end{array}$ & $\begin{array}{l}\text { I: Guidelines C: } \\
\text { No intervention }\end{array}$ & & $\begin{array}{l}\text { Preintervention hospital acquired infection } \\
\text { rates (crude) } 8.9 \%, 10.5 \% \text { Postintervention } \\
\text { hospital aquired infection rates (crude) } 7 \% \text {, } \\
7.8 \%, 6.2 \%, 6 \%, 5.6 \% \mathrm{p}<0.0001 \\
\text { Preintervention hospital aquired infection } \\
\text { rates (adjusted) } 8.5 \%, 9.9 \% \text { Postintervention } \\
\text { hospital aquired infection rates (adjusted) } \\
6.7 \%, 7.5 \%, 6.7 \%, 6.0 \%, 6.2 \% \mathrm{p}<0.0001 \\
\text { Hospital acquired urinary tract infection: } \\
3.1 \%, 3.8 \% 2.8 \%, 3.0 \%, 2.1 \%, 1.0 \%, 2.0 \% \\
\mathrm{p}=0.00083 .2 \%, 3.1 \%, 2.6 \%, 2.9 \%, 2.3 \% \text {, } \\
1.9 \%, 2.3 \% \mathrm{p}=0.032\end{array}$ \\
\hline $\begin{array}{l}\text { Mitchell } \\
\text { and Jones } \\
1996^{32} \text { ITS } \\
\text { Australia }\end{array}$ & $\begin{array}{l}\text { Nurses (number } \\
\text { not specified) on } \\
1 \text { medical ward in } \\
\text { an acute care } \\
\text { hospital }\end{array}$ & Falls & $\begin{array}{l}\text { I: Protocol C: No } \\
\text { intervention }\end{array}$ & & $\begin{array}{l}\text { Fall rates reduced from } 7.8 \text { per } 1000 \text { bed } \\
\text { days before intervention to } 4.4 \\
\text { postintervention } p=0.06\end{array}$ \\
\hline $\begin{array}{l}\text { Tilden } \\
1987^{33} \text { ITS } \\
\text { US }\end{array}$ & $\begin{array}{l}22 \text { Nurses in } 1 \\
\text { hospital } \\
\text { emergency } \\
\text { department }\end{array}$ & $\begin{array}{l}\text { Female adult } \\
\text { trauma }\end{array}$ & $\begin{array}{l}\text { I: Guidelines; } \\
\text { staff education } \\
\text { programme; local } \\
\text { consensus } \\
\text { processes C: No } \\
\text { intervention }\end{array}$ & $\begin{array}{l}\text { Increase in rate of identification of positive } \\
\text { battering from } 7 \text { cases }(9.72 \% \text { of total) to } \\
17 \text { cases }(22.97 \% \text { of total }) p=0.03 \text { OR: } \\
\text { likelihood of identification } 2.74 \text { times } \\
\text { greater post-intervention }\end{array}$ & \\
\hline
\end{tabular}


Comparison tables (continued)

\begin{tabular}{|c|c|c|c|c|c|}
\hline $\begin{array}{l}\text { Study and } \\
\text { study design }\end{array}$ & Participants & Clinical area & $\begin{array}{l}\text { Results } \\
\text { comparisons }\end{array}$ & Main process effect & Main patient outcomes \\
\hline \multicolumn{6}{|c|}{ Skill-substitution: } \\
\hline $\begin{array}{l}\text { Greenfield } \\
\text { et al } 1975^{26} \\
\text { RCT-Pt } \\
\text { (Cross over } \\
\text { trial) US }\end{array}$ & $\begin{array}{l}1 \text { nurse and } 13 \\
\text { doctors in } 1 \text { clinic } \\
\text { Patients: } 151 \\
\text { patients } \\
\text { randomised; } 146 \\
\text { analysed: } 76 \\
\text { intervention } 70 \\
\text { control } \\
\text { Proportion of } \\
\text { patients followed } \\
\text { up: } 99 \% \text { in total }\end{array}$ & $\begin{array}{l}\text { Management } \\
\text { of dysuria, } \\
\text { frequency, } \\
\text { and vaginal } \\
\text { discharge }\end{array}$ & $\begin{array}{l}\text { I: Protocol } \\
\text { (nurses) C: No } \\
\text { intervention } \\
\text { (doctors) }\end{array}$ & $\begin{array}{l}\text { Concordance between nurse and } \\
\text { physician on medical history: } 139 / 146 \\
(95.2 \%) 95 \% \text { CI }(91.6 \%-98.7 \%) \\
\text { Concordance on results of physical } \\
\text { examination: } 137 / 146(93.8 \%) 95 \% \text { CI } \\
(89.8 \%-97.7 \%) \text { Concordance on therapy } \\
\text { and referral: } 144 / 146(99 \%) \text { All referrals } \\
\text { appropriate; protocol did not fail to } \\
\text { recommend referral where there should } \\
\text { have been one Laboratory agreed with } \\
\text { nurse in }(93 \%) 54 / 58 \text { urinalyses } 95 \% \text { CI } \\
93.1 \%(86.5 \%-99.6 \%)\end{array}$ & $\begin{array}{l}\text { Alleviation or improvement of symptoms: I: } \\
(97 \%) 66 / 68 \text {; C: }(97 \%) 63 / 65 p=1.00 \text { Results } \\
\text { of treatment with antibiotics (urine samples } \\
\text { sterile): I: }(71 \%) 10 / 14 ; \text { C: }(53 \%) 8 / 15 \\
p=0.45\end{array}$ \\
\hline $\begin{array}{l}\text { Greenfield } \\
\text { et al } 1975^{25} \\
\text { RCT-Pt US }\end{array}$ & $\begin{array}{l}5 \text { nurses and } 32 \\
\text { doctors in } 1 \text { walk } \\
\text { in clinic Patients: } \\
592 \text { randomised } \\
419 \text { completed } \\
\text { study; } 221 \\
\text { Intervention, } 197 \\
\text { Control } \\
\text { Proportion of } \\
\text { patients followed } \\
\text { up: } 70 \% \text { in total }\end{array}$ & $\begin{array}{l}\text { Management } \\
\text { of low back } \\
\text { pain }\end{array}$ & $\begin{array}{l}\text { I: Protocol } \\
\text { (nurses) C: No } \\
\text { intervention } \\
\text { (doctors) }\end{array}$ & $\begin{array}{l}\text { Number of } x \text { ray films ordered: I: } 21 ; \mathrm{C} \text { : } \\
36 \mathrm{p}<0.01 \text { Initial or final diagnosis: no } \\
\text { significant differences except in initial } \\
\text { diagnosis of other (I: } 10.8 \% \text {; }: 1 \% \text { ) } \\
\text { p }<0.01\end{array}$ & $\begin{array}{l}\text { I patients significantly more satisfied than C } \\
\text { patients p<0.005 Symptom relief: resolved or } \\
\text { improved in I: } 71 \%(144 / 203) \text {; C: } 74.6 \% \\
(144 / 193) \text { p=0.43 Complications: no patient } \\
\text { managed by nurses alone developed serious } \\
\text { complications Return with back problems } \\
\text { within } 3 \text { months: I: } 27 \% \text {; C: } 18 \% p=0.04\end{array}$ \\
\hline $\begin{array}{l}\text { Greenfield } \\
\text { et al } 1976^{27} \\
\text { RCT-Pt US }\end{array}$ & $\begin{array}{l}5 \text { Nurses and } 32 \\
\text { doctors in } 1 \text { walk } \\
\text { in clinic Patients: } \\
203 \text { intervention } \\
193 \text { control } \\
\text { Proportion of } \\
\text { patients followed } \\
\text { up: } 72.6 \% \text { in total }\end{array}$ & $\begin{array}{l}\text { Management } \\
\text { of headache }\end{array}$ & $\begin{array}{l}\text { I: Protocol } \\
\text { (nurses) C: No } \\
\text { intervention } \\
\text { (doctors) }\end{array}$ & $\begin{array}{l}\text { Final diagnosis: diagnosis of muscle } \\
\text { headache: I: } 62.1 \%(126 / 203) \text {; C: } 43.5 \% \\
\text { (84/193) p<0.001 Prescription of } \\
\text { medication: no significant differences } \\
\text { except for prescription of minor } \\
\text { tranquillisers: I: } 6.4 \%(13 / 203) \text {; C: } 15.5 \% \\
(30 / 193) \text { p }<0.05\end{array}$ & $\begin{array}{l}\text { Symptom relief: resolved or improved: I: } 73 \% \\
(162 / 222) \text {; C: } 68.5 \%(135 / 197) \mathrm{p}=0.33 \\
\text { Patient satisfaction: satisfaction with } 6 \text { of } 9 \\
\text { elements significantly higher in intervention } \\
\text { group; no significant difference for remaining } \\
3 \text { elements; p ranged from }<0.02 \text { to } \\
<0.00001 \text { for these } 6 \text { elements Development } \\
\text { of serious sequelae: no instances of serious } \\
\text { disease and no admissions to hospital related } \\
\text { to headache in either group }\end{array}$ \\
\hline $\begin{array}{l}\text { Jewell and } \\
\text { Hope } 1988^{18} \\
\text { RCT-Pt UK }\end{array}$ & $\begin{array}{l}1 \text { Nurse and } 1 \\
\text { doctor in } 1 \\
\text { general practice } \\
\text { Patients: } 17 \\
\text { intervention } 19 \\
\text { control } \\
\text { Proportion of } \\
\text { patients followed } \\
\text { up: } 88 \% \\
\text { intervention } \\
100 \% \text { control }\end{array}$ & Hypertension & $\begin{array}{l}\text { I1: Protocol } \\
\text { (nurses) I2: } \\
\text { Protocol } \\
\text { (doctors) }\end{array}$ & $\begin{array}{l}\text { Percentage of patients with record of: } \\
\text { Smoking habit (once): I1: (100\%) 15; I2: } \\
\text { (84\%) 16 patients p=0.32 Urine test } \\
\text { (once): I1: ( } 93 \%) 14 ; \mathrm{I} 2:(21 \%) 4 \text { patients } \\
\text { p<0.00002 Blood pressure (at each visit): } \\
\text { I1: (100\%) 90; I2: (100\%) 103 visits } \\
\text { p=1.00 Pulse (at each visit): I1: (98\%) } 88 \text {; } \\
\text { I2: (65\%) } 67 \text { visits p<0.01 Weight (at each } \\
\text { visit): I1: (90\%) 81; I2: (37\%) } 38 \text { visits } \\
\text { p<0.001 }\end{array}$ & 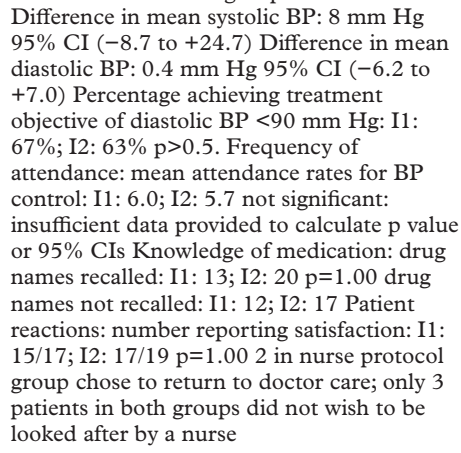 \\
\hline $\begin{array}{l}\text { Klassen et } \\
\text { al } 1993^{28} \\
\text { RCT-Pt } \\
\text { Canada }\end{array}$ & $\begin{array}{l}21 \text { Triage nurses } \\
\text { and doctors } \\
\text { (number not } \\
\text { specified) in } 1 \\
\text { emergency room } \\
\text { Patients: } 491 \\
\text { intervention } 494 \\
\text { control } \\
\text { Proportion of } \\
\text { patients followed } \\
\text { up: } 98.9 \%\end{array}$ & $\begin{array}{l}\text { Radiology } \\
\text { referrals }\end{array}$ & $\begin{array}{l}\text { I: Protocol } \\
\text { (nurses) C: No } \\
\text { intervention } \\
\text { (doctors) }\end{array}$ & $\begin{array}{l}\text { Percentage of patients having } x \text { ray films } \\
\text { ordered: I: } 81.9 \% \text {; }: 87.1 \% p=0.03 \\
\text { Positive findings as percentage of ordered } \\
x \text { ray films: I: } 40.8 \% \text {; }: 42.6 \% p=0.21 \\
\text { Missed positive findings as percentage of } \\
\text { whole group: I: } 3.2 \% \text {; }: 0 \% p<0.001 \\
\text { Mean time spent in the emergency } \\
\text { department: I: } 3.3 \text { hours; C: } 3.6 \text { hours } \\
\text { p }<0.001\end{array}$ & \\
\hline $\begin{array}{l}\text { Zeler et al } \\
1992^{19} \\
\text { RCT-Pt } \\
\text { Australia }\end{array}$ & $\begin{array}{l}\text { Nurses and } \\
\text { doctors (numbers } \\
\text { not specified) } \\
\text { working in } 1 \\
\text { cardiothoracic } \\
\text { intensive care unit } \\
\text { Patients: } 159 \\
\text { intervention } 163 \\
\text { control } \\
\text { Proportion of } \\
\text { patients followed } \\
\text { up: } 67 \% \\
\text { intervention } 91 \% \\
\text { control }\end{array}$ & $\begin{array}{l}\text { Management } \\
\text { of } \\
\text { postoperative } \\
\text { bleeding after } \\
\text { cardiac } \\
\text { surgery }\end{array}$ & $\begin{array}{l}\text { I: Guideline } \\
\text { (nurses) C: No } \\
\text { intervention } \\
\text { (doctors) }\end{array}$ & $\begin{array}{l}\text { Number of patients in whom coagulation } \\
\text { tests performed: I: } 13 \%(14 / 108) \text {; C: } 14 \% \\
\text { (20/146) p=0.40 Mean time to } \\
\text { coagulation test: I: } 291 \text { minutes; C: } 454 \\
\text { minutes p=0.30 Volume of packed cells } \\
\text { infused: presented graphically p<0.01 } \\
\text { Volume of total blood and blood products } \\
\text { infused: presented graphically } p<0.05\end{array}$ & $\begin{array}{l}\text { Mean postoperative blood loss: I: } 817 \mathrm{ml} \text {; C: } \\
976 \mathrm{ml} \text { p=0.08 Mean activated partial } \\
\text { thromboplastin time: I: } 31.7 \mathrm{~s} \text {; }: 47.3 \mathrm{~s} \\
\mathrm{p}=0.01 \text { Number requiring emergency } \\
\text { sternotomy: I: } 4 \%(4 / 108) \text {; C: } 8 \%(12 / 146) \\
p=0.32\end{array}$ \\
\hline
\end{tabular}

RCT-Pr=randomised controlled trial by provider; RCT-Pt=randomised controlled trial by patient; CBA=controlled before and after study; ITS $=$ interrupted time series study; I=intervention; I1, I2, I3=intervention 1, intervention 2, intervention 3; C=control; LDL-cholesterol=low density lipoprotein cholesterol; HDL-cholesterol=high density lipoprotein cholesterol; $\mathrm{RR}=$ risk ratio; $\mathrm{BP}=$ blood pressure; $\mathrm{OR}=$ odds ratio.

and lectures; group two received additional provider and patient education at each clinic visit; and group three received an additional protocol. This was a complex intervention, comprising a flowchart record incorporating the recommendations of the guideline - that is, a patient specific prompt - and a redefinition of the nurse's role. Herman et $a l^{16}$ found that an 
active dissemination strategy (involving provider education) had a minor effect on professional behaviour in the group receiving only guidelines, educational materials, and lectures. The impact of the guidelines on outcomes of care was also assessed: a significant difference between intervention and control groups was found, but in the opposite direction to that which was expected: the percentage of patients declining immunisation increased in the intervention period to $13.2 \%$ in the prevention team group, $5.8 \%$ in the patient education group, and $5.9 \%$ in the control group. However, there was an error in the unit of analysis in this study: hence findings were more likely to be significant.

McDonald et $a l^{17}$ compared the effect of computerised prompts specific to the patient at the time of consultation, computerised prompts plus bibliographic citations, and standard care on the use and follow up of medications. However, the results for nurses were presented with the two intervention groups combined, so it is not possible to compare the two methods of implementation. With the computerised prompts and computerised prompts plus citations groups combined, the intervention did not seem to lead to a significant change in nurses' behaviour; this finding was contrary to that for physicians in the same study. It is possible that this negative finding was due to a lack of power to detect a difference ( $\mathrm{n}=$ five nurses).

GUIDELINE VERSUS NO GUIDELINE STUDIES

Nine studies (table) compared the introduction of guidelines with a no guideline control. ${ }^{20-22} 24{ }^{29-33}$ These studies aimed to improve the quality of care delivered by the target profession. Of these, four were randomised controlled trials, ${ }^{212429} 30$ three were interrupted time series studies, ${ }^{20} 3233$ and two were controlled before and after studies. ${ }^{2231}$ Targeted behaviours were nutrition therapy for patients with non-insulin dependent diabetes, ${ }^{21}$ management of labour, ${ }^{24}$ discharge planning, ${ }^{29}{ }^{30}$ management of intravenous therapy, ${ }^{31}$ lipid lowering, ${ }^{22}$ identification of battered women, ${ }^{33}$ infection control, ${ }^{20}$ and reduction in rates of falls. $^{32}$

Five studies assessed the effect of guidelines in terms of the process of care. In four of these, significant changes in at least some of the assessed processes of care were identified. $^{22} 243133$ For example, the study of Frigoletto et $a l^{24}$ of the management of labour by nurse midwives found significant differences in practices relating to the management of labour between the active management protocol and usual care groups. In the active management group, the frequency of vaginal examinations was significantly higher (mean interval 1.6 hours $v 2.5$ hours for standard care group); significantly more women received oxytocin to hasten labour $(70 \% v 56 \%)$ and significantly fewer women requested and received epidural anaesthesia ( $54 \% v 64 \%)$. The analysis reported by Larson and Hargiss ${ }^{31}$ is almost certainly inappropriate, but insufficient data were provided to be certain: there is no explanation as to how the dependent variable was calculated for the analysis of variance (ANOVA), and clustering of the data has probably not been taken into account.

Eight studies measured outcomes of care. In six of these, significant differences in favour of the intervention group were found for at least some outcomes of care. ${ }^{20-22} 2429{ }^{30}$ However, the findings from several of these studies were equivocal, with no significant change being noted in other outcome variables assessed. For some studies, the observed change in what seemed to be the primary outcome measure was non-significant. ${ }^{21243032}$

Only one study ${ }^{21}$ included a formal economic evaluation. This study assessed the effect of medical nutrition therapy for patients with non-insulin dependent diabetes administered according to practice guidelines with medical nutrition therapy administered in line with standard care. Findings were suggestive of a cost efficiency advantage in favour of the control group when one outcome measure (glycated haemoglobin) was considered, but no difference when an alternative outcome measure (fasting plasma glucose concentration) was examined. The studies described by Naylor et $a l,{ }^{2930}$ evaluating the effects of a protocol for discharge planning compared with standard discharge procedures, and Larson and Hargiss, ${ }^{31}$ evaluating the effectiveness of specially trained nurses who used guidelines in the maintenance of intravenous treatment compared with standard care, suggested that direct healthcare costs are decreased by care driven by a guideline, but these authors did not provide sufficient data to ascertain whether these cost savings were offset by the cost of guideline dissemination and implementation.

SKILL SUBSTITUTION STUDIES

In the remaining six studies (table) ${ }^{18}{ }^{19} 25-28$ the performances of nurses operating in accordance with a guideline were compared with standard care, generally provided by a physician. All six studies were randomised controlled trials. The aim was skill substitution, generally in the interests of greater efficiency. Targeted behaviours were: management of dysuria, frequency and vaginal discharge ${ }^{26}$ management of low back pain, ${ }^{25}$ management of headache, ${ }^{27}$ management of hypertension, ${ }^{18}$ referral for $x$ ray examination, ${ }^{28}$ and management of postoperative bleeding after cardiac surgery. ${ }^{19}$ Study settings were: outpatients, ${ }^{25} 26$ inpatients, ${ }^{19}$ the emergency room, ${ }^{28}$ and primary care. ${ }^{18} 28$

In these studies, a finding of no significant difference, or of a positive effect in favour of the nurse or other professional allied to medicine, was appropriate. Findings from all six studies which examined the processes and the five which examined outcomes of care generally supported the hypothesis of no difference. However, appropriate sample size calculations were not reported in any study; thus we cannot be sure that there was sufficient power to detect a clinically relevant difference. The finding of no significant difference may therefore have been due to small sample size. Clearly this is 
weak evidence, as there was no comparison with patients cared for by nurses not using guidelines.

\section{Discussion}

Studies which compared different dissemination and implementation strategies suggest educational interventions are of value in the dissemination of guidelines, and confer a benefit over passive dissemination approaches. This finding is in line with that of Grimshaw and Russell for physicians. ${ }^{2}$ However, from the limited evidence presented in the two studies in which there were positive changes in process or outcomes, ${ }^{16}{ }^{23}$ we cannot tell which forms of education are most effective in bringing about changes in behaviour. Grimshaw and Russell ${ }^{2}$ suggested that educational strategies requiring more active participation, such as educational outreach visits and targeted seminars, are more likely to lead to changes in behaviour among physicians; there is an urgent need to investigate whether this assertion holds true for nurses, midwives, and other professions allied to medicine. By contrast, a computerised prompt specific to the patient at the time of consultation $^{17}$ did not seem to alter nurses' behaviour, despite evidence that this is an effective way of changing the behaviour of physicians. ${ }^{34}{ }^{35}$ However, this finding was based on a small sample of nurses: further research is required before this implementation strategy can be rejected.

Interventions evaluated by Herman et $a l^{16}$ and by Larson and Hargiss, ${ }^{31}$ were complex; complex interventions were also described by French et $a l^{20}$ and by Mitchell and Jones. ${ }^{32}$ Wensing and $\mathrm{Grol}^{36}$ have shown that multiple interventions are more likely to be effective in bringing about behavioural change than are single interventions, especially if multiple interventions involve individual instruction, feedback, and reminders. Findings from studies by Herman et $a l^{16}$ and Larson and Hargiss ${ }^{31}$ provide some evidence of the impact of multiple interventions. However, further research, comparing single and multiple interventions in the same population, is required before we can be sure that the findings of Wensing and $\mathrm{Grol}^{36}$ are applicable to nursing and professions allied to medicine.

Findings from the 18 studies identified provide some evidence that care driven by guidelines can be effective in changing the process and outcome of care. However, some caution is needed in interpreting the findings from our review, and in generalising these findings to other professions and settings.

Most studies were carried out in a single setting, focused on only one profession, and often involved very few health professionals (in several cases, only one nurse). The possibility that the findings were specific to the setting or to the individual people cannot be ruled out. In some cases, the health professionals who were studied were selected specifically because of their enthusiasm or expertise $;^{21}$ the guidelines may have been less effective in the hands of less committed or less highly qualified or experienced staff. As many of the authors themselves recognise, ${ }^{22} 242731$ most of the studies were threatened by the Hawthorne effect, as health professionals were aware that their performance was being assessed. The timing of the assessment of process and outcome may also have had an impact: studies we identified did not, in general, provide sufficient information to assess whether any changes in performance found were sustained after the initial impetus of guideline dissemination.

Only three of the 18 studies provided evidence from randomised controlled trials in which the units of randomisation and analysis were the health professional, or from crossover trials where all health professionals experienced each level of intervention. In studies in which the units of randomisation and analysis were the patients, and where the same health professionals provided care for patients in the intervention and control groups, there is the possibility of contamination of the control group by carry over of knowledge of the guideline. Franz et $a l^{1}$ explicitly recognised that this may have occurred in their study and may be a reason for the lack of significant findings in the primary outcome measures. In controlled before and after studies, bias may be introduced if the baseline characteristics of the sites are different, or if there is some other systematic difference between sites. Shaffer and Wexler ${ }^{22}$ recognised that bias may have been introduced by the fact that allocation to the intervention and control groups was not random, but rather was at the discretion of the patients' regular physicians. In time series designs, there is a risk of contemporaneous changes, a possibility recognised by Tilden and Shepherd. ${ }^{33}$

Many of the studies fall short of the quality criteria for randomised controlled trials, controlled before and after studies, and interrupted time series studies set down by EPOC. Important methodological details were often not reported-for example, few studies specified initially the size of difference in process or outcome that would be considered to be "clinically significant" and reported whether sample sizes were calculated accordingly. Thus we cannot be sure that the studies were adequately powered to detect a clinically important difference. Similarly, in the studies seeking to show no significant difference between care provided by a nurse and driven by a guideline and standard care provided by a physician, appropriate sample size calculations were not reported. In these studies, the finding of no significant difference may have been due to small sample size. Pocock ${ }^{37}$ advises that, in general, larger sample sizes are required when the aim is to infer that there is no difference.

Potential sources of bias include a lack of blinding to allocation. If data collectors were aware of which patients were in the intervention and control groups, data collection procedures may have been biased in favour of the intervention group. Few studies reported an intention to treat analysis, although there was often substantial (and, in some cases, differential) attrition from intervention and control samples. 
It is mostly impossible to tell whether the guidelines evaluated were based on evidence. Although many were based on a literature review, the extent to which these reviews were systematic was not described, nor were the quality criteria by which any evidence was assessed. The information contained in the papers does not allow us to state whether this lack of an evidence base was due to failure to search for the evidence systematically, or whether there was virtually none. This calls into question the validity of the guidelines.

More research is clearly required in the development, dissemination, implementation, evaluation, and cost effectiveness of clinical guidelines as a strategy for improving professional practice in nursing and professions allied to medicine. Only then will a decision be possible on whether their potential for improving the practice of nurses and professions allied to medicine is as great as it is for doctors. ${ }^{1}$

We thank the NHS research programme "evaluating methods to promote the implementation of research findings" for funding
this project. We acknowledge the helpful comments and advice this project. We acknowledge the helpful comments and advice
of members of the EPOC Advisory Board and our three exterof members of the EPOC Advisory Board and our three external peer reviewers, Philip Ayres, Ellen Hodnett, and Mary Ann
Thomson. We also thank Carol Riccalton, a freelance Thomson. We also thank Carol Riccalton, a freelance information scientist, and Julie Glanville, from the NHS Centre for Reviews and Dissemination at York, for help with searching. A big thank panel who assisted in the development of the protocol: Martin Eccles, Ashley Adamson, Pam Dawson, Nick Miller, Christine Bond, Don Lorimer, Soraya Meah, Rita Bell, Karen Jones, Rick Telford, and Roger Paxton. We thank Trevor Sheldon and two anonymous reviewers for helpful comments on an earlier draft.

1 Grimshaw JM, Russell IT. Effect of clinical guidelines on medical practice: a systematic review of rigorous evaluations. Lancet 1993;342:1317-22.

2 Effective Health Care. Implementing clinical practice guidelines. Leeds: University of Leeds, 1994.

3 Von Degenberg K, Deighan M. Guideline development: a model of multi-professional collaboration. In: Deighan $M$, Hitch S, eds. Clinical effectiveness from guidelines to cost-effective practice. London: Department of Health, 1995;

4 Bond CM, Grimshaw JM. Multidisciplinary guideline development: a case study from community pharmacy.

5 Cheater FM, Closs SJ. The effectiveness of methods of dissemination and implementation of clinical guidelines for nursing practice: a selective review. Clinical Effectiveness in Nursing 1997;1:4-15.

6 McClarey M. Identifying priorities for guideline development as a result of nursing needs. Dynamic Quality Initiative Network News 1997;6:4-5.

7 Lomas J. Words without action: the production, dissemination and impact of consensus recommendations. Annu Rev Public Health 1991;12:41-65.

8 Davis DA, Thomson MA, Oxman AD, et al. Changing physician performance: a systematic review of the effect of continuing medical education strategies. $\mathscr{F} A M A$ 1995;274: $700-5$

9 Lomas J, Haynes RB. A taxonomy and critical review of tested strategies for the application of clinical practice recommendations: from official to individual clinical policy [review]. Am f Prev Med 1988;4(suppl):77-94.

10 Grol R, Thomas S, Roberts R. Development and implementation of guidelines for family practice: lessons from The tation of guidelines for family practice: lessons from
Netherlands [editorial]. $\mathcal{F}$ Fam Pract 1995;40:435-9.

11 Grol R. Implementing guidelines in general practice care. Quality in Health Care 1992;1:184-91.
12 Thomas L, McColl E, Cullum N, et al. Systematic review of the effectiveness of guidelines in nursing, midwifery and professions allied to medicine. Final report. Newcastle upon Tyne: University of Newcastle upon Tyne, Centre for Health Services Research, 1998

13 Grimshaw J, Freemantle N, Wallace S, et al. Developing and mplementing clinical practice guidelines. Quality in Health Care 1995;4:55-64.

14 Field MJ, Lohr KN. Clinical practice guidelines: directions for a new program. Washington: National Academy Press, 1990.

15 Dunford J, Smith K, Nunn R. Blackwell idealist user's guide. Oxford: Blackwell Science, 1995.

16 Herman CJ, Speroff T, Cebul RD. Improving compliance with immunization in the older adult: results of a randomized cohort study. F Am Geriatr Soc 1994;42:11549.

17 McDonald CJ, Wilson GA, McCabe GP. Physician response to computer reminders. $\mathcal{F} A M A \quad 1980 ; 244: 1579-81$.

18 Jewell D, Hope J. Evaluation of a nurse-run hypertension clinic in general practice. Practitioner 1988;232:484-7.

19 Zeler KM, McPharlane TJ, Salamonsen RF. Effectiveness of nursing involvement in bedside monitoring and control of coagulation status after cardiac surgery. Am 7 Crit Care 1992;1:70-5.

20 French GL, Cheng AF, Wong SL, et al. Repeated prevalence surveys for monitoring effectiveness of hospital infection control. Lancet 1989;ii:1021-3.

21 Franz MJ, Monk A, Barry B, et al. Effectiveness of medical nutrition therapy provided by dietitians in the management of non-insulin-dependent diabetes mellitus: a randomized,
controlled clinical trial. f Am Diet Assoc 1995;95:1009-17.

22 Shaffer J, Wexler LF. Reducing low-density lipoprotein cholesterol levels in an ambulatory care system. Results of a multidisciplinary collaborative practice lipid clinic compared with traditional physician-based care. Arch Intern Med 1995;155:2330-5.

23 Seto WH, Ching TY, Yuen KY, et al. The enhancement of infection control in-service education by ward opinion leaders. Am $\mathcal{F}$ Infect Control 1991;19:86-91.

24 Frigoletto FD, Lieberman E, Lang JM, et al. A clinical trial of active management of labor [published erratum appears 1995;333:745-50.

25 Greenfield S, Anderson H, Winickoff RN, et al. Nurseprotocol management of low back pain. Outcomes, patient protocol management of low back pain. Outcomes, patient 1975;123:350-9.

26 Greenfield S, Friedland G, Scifers S, et al. Protocol management of dysuria, urinary frequency, and vaginal discharge f Fam Pract 1975;2:179-84.

27 Greenfield S, Komaroff AL, Anderson H. A headache protocol for nurses: effectiveness and efficiency. Arch Intern Med 1976;136:1111-6.

28 Klassen TP, Ropp LJ, Sutcliffe T, et al. A randomized, controlled trial of radiograph ordering for extremity trauma in a pediatric emergency department. Ann Emerg Med 1993;22:1524-9.

29 Naylor MD. Comprehensive discharge planning for hospitalized elderly: a pilot study. Nurs Res 1990;39:156-61.

30 Naylor M, Brooten D, Jones $\mathrm{R}$, et al. Comprehensive discharge planning for the hospitalized elderly. A randomized clinical trial. Ann Intern Med 1994;120:999-1006.

31 Larson E, Hargiss C. A decentralized approach to maintenance of intravenous therapy. Am $\mathcal{F}$ Infect Control 1984;12:177-86.

32 Mitchell A, Jones N. Striving to prevent falls in an acute care setting - action to enhance quality. 7 Clin Nurs 1996;5:213-

33 Tilden VP, Shepherd P. Increasing the rate of identification of battered women in an emergency department: use of a nursing protocol. Res Nurs Health 1987;10:209-15

34 McDonald CJ, Hui SL, Smith DM, et al. Reminders to physicians from an introspective computer medical record. Ann Intern Med 1984;100:130-8.

35 Johnston ME, Langton $\mathrm{KB}$, Haynes RB, et al. Effects of computer-based clinical decision support systems on clinician performance and patient outcome: a critical appraisal of research. Arch Intern Med 1994;120:135-42.

36 Wensing $M$, Grol R. Single and combined strategies for implementing changes in primary care: a literature review. Int $\mathcal{f}$ Qual Health Care 1994;6:115-32.

37 Pocock SJ. Clinical trials: a practical approach. Chichester: John Wiley, 1983. 